\title{
Valuing modular nuclear power plants in finite time decision horizon
}

\author{
Shashi Jain ${ }^{\mathrm{a}, \mathrm{b}, *, 1}$, Ferry Roelofs ${ }^{\mathrm{b}}$, Cornelis W. Oosterlee ${ }^{\mathrm{a}, \mathrm{c}}$ \\ a TU Delft, Delft Institute of Applied Mathematics, Delft, the Netherlands \\ ${ }^{b}$ Nuclear Research Group, Petten, the Netherlands \\ c CWI-Centrum Wiskunde E Informatica, Amsterdam, the Netherlands
}

\section{A R T I C L E I N F O}

\section{Article history:}

Received 7 May 2012

Received in revised form 6 November 2012

Accepted 9 November 2012

Available online 17 November 2012

\section{JEL classification:}

D81

Q40

G11

\section{Keywords:}

Real option

Stochastic grid method

Modularity

Nuclear power plants

Finite time decision horizon

\begin{abstract}
A B S T R A C T
Small and medium sized reactors, SMRs, (according to IAEA, 'small' refers to reactors with power less than $300 \mathrm{MWe}$, and 'medium' with power less than $700 \mathrm{MWe}$ ) are considered as an attractive option for investment in nuclear power plants. SMRs may benefit from flexibility of investment, reduced upfront expenditure, enhanced safety, and easy integration with small sized grids. Large reactors on the other hand have been an attractive option due to the economy of scale. In this paper we focus on the economic impact of flexibility due to modular construction of SMRs. We demonstrate, using real option analysis, the value of sequential modular SMRs. Numerical results under different considerations of decision time, uncertainty in electricity prices, and constraints on the construction of units, are reported for a single large unit and for modular SMRs.
\end{abstract}

(c) 2012 Elsevier B.V. All rights reserved.

\section{Introduction}

Deregulation of the electricity market has been driven by the belief in increased cost-efficiency of competitive markets. There is a need for valuation methods to make economic decisions for investment in power plants in these uncertain environments. Kessides (2010) emphasizes the use of real options analysis (ROA) to estimate the option value that arises from the flexibility to wait and choose between further investment in the nuclear plant and other generating technologies as new information emerges about energy market conditions.

There is an increased interest in SMRs as an alternative to large Gen III type nuclear reactors (Boarin et al., 2012). This is primarily because the former has, amongst other benefits, comparatively low upfront costs and flexibility of ordering due to its modular nature (Carelli et al., 2010). When comparing economy of large reactors and SMRs, it's necessary to take into account the value of flexibility arising due to modular construction, which traditional valuation methods like NPV cannot. As the decisions to order new reactors would be planned for finite time horizons, there is a need to adapt the real option valuation for modular construction, as proposed by Gollier et al. (2005), to a finite time horizon. The case studies presented here are not only important for the

\footnotetext{
* Corresponding author.

E-mail addresses: s.jain@cwi.nl (S. Jain), roelofs@nrg.eu (F. Roelofs), c.w.oosterlee@cwi.nl (C.W. Oosterlee).

1 Thanks to CWI-Centrum Wiskunde \& Informatica, Amsterdam.
}

construction of power plants but they are also relevant for a larger class of decision questions in which flexibility due to modularity and economy of scale plays an important role.

The real options approach for making investment decisions in projects with uncertainties, pioneered by Arrow and Fischer (1974), Henry (1974), Brennan and Schwartz (1985) and McDonald and Siegel (1986) became accepted in the past decade. Dixit and Pindyck (1991) and Trigeorgis (1996) comprehensively describe the real options approach for investment in projects with uncertain future cash flows. Using real options it's possible to value the option to delay, expand or abandon a project with uncertainties, when such decisions are made following an optimal policy.

ROA has been applied to value real assets like mines (Brennan and Schwartz (1985)), oil leases (Paddock, et. al (1988)), patents and R\&D (Lucia and Schwartz (2002)). Pindyck (1993) uses real options to analyze the decisions to start, continue or abandon the construction of nuclear power plants in the 1980's. He considers uncertain costs of a reactor rather than expected cash flows for making the optimal decisions. Rothwell (2006) uses ROA to compute the critical electricity price at which a new advanced boiling water reactor should be ordered in Texas.

In this paper we focus on the value of flexibility that arises from the modular construction of SMRs. Our approach is similar to Gollier et al. (2005), where the firm needs to make a choice between a single high capacity reactor (1200 MWe) or a flexible sequence of modular SMRs $(4 \times 300 \mathrm{MWe})$. We, however, consider finite time horizon before which the investment decision should be made. In a competitive market the 


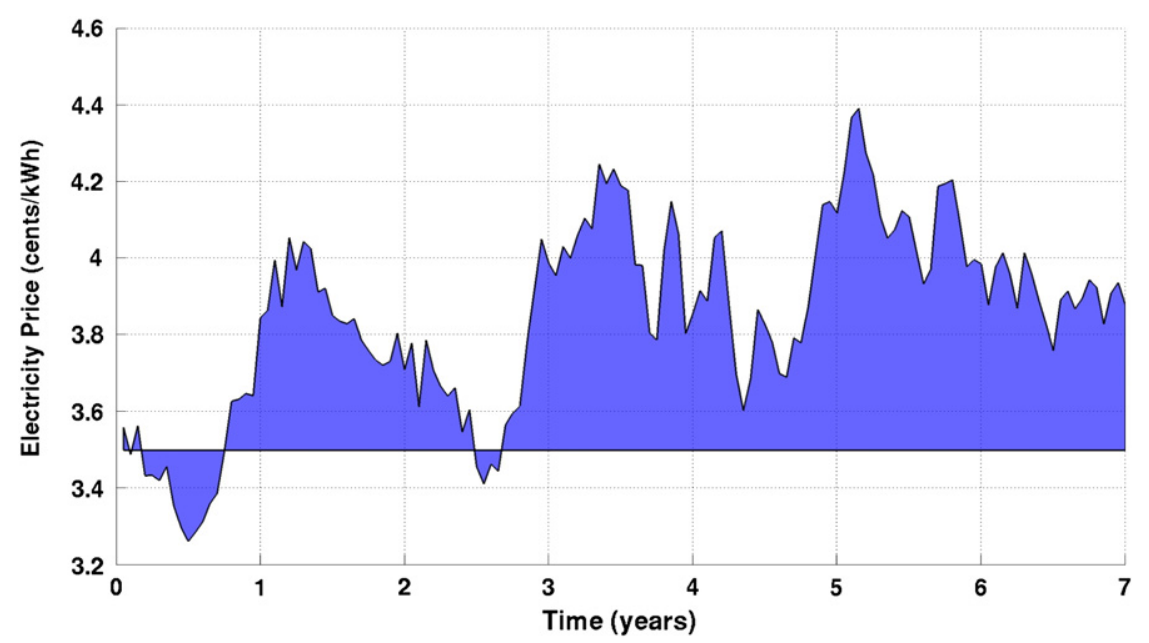

Fig. 1. The area between the electricity path (starting at 3.5 cents $/ \mathrm{kWh}$ ) and cost of operation $=3.5$ cents $/ \mathrm{kWh}$, gives cash flow for the reactor.

firms cannot delay an investment decision for ever and need to decide before the anticipated entry of a competitor, or before a technology becomes obsolete. Also utilities need to meet the electricity demand with some minimum reliability, which restricts their decision horizon to finite time. The investment rules, such as the optimal time to start construction and the real option value of the investment, can differ significantly with changing decision horizons.

Real options can be priced with methods used for pricing American- or Bermudan-style financial options. We use a simulation based algorithm, called the stochastic grid method (SGM) (Jain and Oosterlee, 2012), for computing the real option values of modular investment decisions. SGM has been used to price Bermudan options in (Jain and Oosterlee, 2012) with results comparable to those obtained using the well-known least squares method (LSM) of Longstaff and Schwartz (2001), but typically with tighter confidence intervals using fewer Monte Carlo paths. The option values are computed by generating stochastic paths for electricity prices, and thus with uncertain future cash flows. As an outcome of computing the real option price, we find the optimal electricity price at which a new module should be ordered.

In the sections to follow we state the problem of modular investment in nuclear power plants and compare it with its counterpart in the financial world. In Section 2 we describe the problem and its real option formulation. In Section 3 the mathematical formulation behind the problem is discussed. Section 4 gives the description of the stochastic grid method used to value the real option. Section 5 describes in detail the application of the method to the nuclear case. Finally, Section 6 gives some concluding remarks and possible future research questions that need to be addressed.

\section{Problem context}

We consider a competitive electricity market where the price of electricity follows a stochastic process. The utility faces the choice of either constructing a single large reactor of $1200 \mathrm{MWe}$, or sequentially constructing four modules of 300 MWe each. The total number of series units is denoted by $n$. Unit number $i$ is characterized by discounted averaged cost per KWh equal to $\theta_{i}$, its construction time is denoted by $C_{i}$ and the lifetime of its operation by $L_{i}$. Both construction and lifetime are expressed in years. It is assumed that different modules are constructed in sequence, where,

1. similar to the case of Gollier et al. (2005), construction of module $i+1$ cannot be decided until construction of unit $i$ is over, i.e. no overlap in construction of modules is allowed.

2. a more relaxed constraint where the construction of unit $i+1$ can be decided from any time subsequent to the start of construction of unit $i$.
We assume a constant discount rate denoted by $r$ here.

The utility here needs to take a decision to start the construction of the modules within a finite time horizon, denoted by $T_{i}$ for the $i$ th module. In terms of financial options, $T_{i}$ represents the expiration time for the 'option to start the construction of the ith module'. Unlike financial options, it's difficult to quantify the expiration time for real options, and it is usually taken as the expected time of arrival of a competitor in the market, or time before which the underlying technology becomes obsolete. In case of an electricity utility, it also represents the time before which the utility needs to set up a plant to meet the electricity demand with certain reliability. ${ }^{2}$

\subsection{The real option formulation}

The problem of modular construction can be formulated as a multiple exercise Bermudan option. In this case we consider the stochastic process, $X_{t}$, to be the process which models the electricity price. The payoff, $h_{i}\left(X_{t}=x\right)$, for the real option problem is the expected net cash flows per unit power of electricity sold through the lifetime of module $i$, when it gets operational at time $t$ and state $X_{t}=x$.

Fig. 1 illustrates the profit from the sale of electricity for one realized electricity price path. The cost of operation, $\theta$, in the illustration is 3.5 cents/kWh and the area between the electricity path and $\theta$ gives the profit from the sale of electricity. We are interested in the expected profit, i.e. the mean profit from all possible electricity paths in the future. This expected profit (or net cash flow) is the payoff, $h_{i}\left(X_{t}\right)$, for the real option.

The revenue, $R_{i}$, for the $i$ th module, for every unit power of electricity sold through its lifetime $L_{i}$, starting construction at time $t$, when the electricity price is $X_{t}=x$, can be written as

$R_{i}\left(X_{t}=x\right)=\mathbb{E}\left[\int_{t+C_{i}}^{t+C_{i}+L_{i}} e^{-r u} X_{u} d u \mid X_{t}=x\right]$

$R_{i}$ is the discounted expected gross revenue over all possible electricity price paths. The revenue starts flowing in once the construction is over, and therefore the range for the integral starts from $t+C_{i}$ and lasts as long as the plant is operational, i.e. until $t+C_{i}+L_{i}$. Similarly, the cost of operating the $i$ th module, $K_{i}$, through its lifetime for every unit power of electricity generated, is:

$K_{i}=\int_{t+C_{i}}^{t+C_{i}+L_{i}} e^{-r u} \theta_{i} d u$

\footnotetext{
${ }^{2}$ Reliability is measured as the probability of the number of unplanned outages in a year with one of the reasons for such an outage being demand exceeding available generation.
} 
Here $\theta_{i}$, the cost of operating the reactor per $\mathrm{kWh}$ is assumed to be constant. Therefore, the net discounted cash flow, for module $i$, is given by:

$h_{i}\left(X_{t}=x\right)=R_{i}\left(X_{t}=x\right)-K_{i}$.

Eqs. (1) to (3) give the expected profit from the sale of electricity through the life of the nuclear reactor.

Eq. (3) is the mean profit from all possible electricity paths in the future.

The expiration time $T_{n}$ is the time before which the last module should be ordered. The optimal exercise policy $\pi=\left\{\tau_{n}, \ldots, \tau_{1}\right\}$, is then defined by the determination of the optimal times for starting the construction of different modules, with $\tau_{i}$, the optimal time for starting the construction of module $i$, so that the net cash flow from the different modules is maximized.

\subsection{Electricity price model}

The uncertain parameter in our pricing model is the electricity price. Modeling electricity spot prices is difficult primarily due to factors like:

- Lack of effective storage, which implies electricity needs to be continuously generated and consumed.

- The consumption of electricity is often localized due to constraints of relatively poor grid connectivity.

- The prices show other features like daily, weekly and seasonal effects, that vary from place to place.

Models for electricity spot prices have been proposed by Pilipovic (1998) and Lucia and Schwartz (2002). Barlow (2002) develops a stochastic model for electricity prices starting from a basic supply/demand model for electricity. These models are focused on the short term fluctuations of electricity prices which help better pricing of electricity derivatives.

As decisions for setting up power plants look at long term evolution of electricity prices, we, like Gollier et al. (2005), use the basic Geometric Brownian Motion (GBM) as the electricity price process. However, it should be noted that within our modeling approach we can easily include other price processes.

\subsubsection{Geometric Brownian motion}

If at any time $t$ the electricity price is given by $X_{t}$ cents $/ \mathrm{kWh}$, then the electricity price process is given by

$d X_{t}=\alpha X_{t} d t+\sigma X_{t} d W_{t}$

where $\alpha$ represents the constant growth rate of $X_{t}, \sigma$ is the associated volatility and $W_{t}$ is the standard Brownian motion. In our model we assume $\alpha$ and $\sigma$ to be constant. A closed form solution to the above SDE can be obtained using Ito's lemma, and is given by:

$X_{t}=X_{0} e^{\left(\left(\alpha-\frac{\sigma^{2}}{2}\right) t+\sigma \sqrt{t} z\right)}$,

where $Z$ is a standard normal variable. Also it can be seen that the above process has a $\log$-normal distribution, i.e. $\log \left(X_{t}\right)$ has a Gaussian distribution with mean

$\mathbb{E}\left[\log \left(X_{t}\right)\right]=\log \left(X_{0}\right)+\left(\alpha-\frac{\sigma^{2}}{2}\right) t$

and variance

$\operatorname{Var}\left(\log \left(X_{t}\right)\right)=\sigma^{2} t$
Table 1

Construction time and discounted averaged costs used for the large reactor and the modular case.

\begin{tabular}{lll}
\hline & $\begin{array}{l}\text { Construction time } \\
\text { (months) }\end{array}$ & $\begin{array}{l}\text { Discounted average cost } \\
\text { (cents/KWh) }\end{array}$ \\
\hline Large reactor & 60 & 2.9 \\
& & \\
Modular case & 36 & 3.8 \\
Module 1 & 24 & 2.5 \\
Modules 2 to 4 & &
\end{tabular}

\section{Mathematical formulation}

The optimal time to order ${ }^{3}$ a new reactor under uncertain electricity price is solved using dynamic programming, where an optimal solution is found recursively moving backwards in time. Here we re-frame the problem stated above as a dynamic programming problem.

\subsection{Dynamic programming formulation}

In order to construct all the modules at the optimal time, using Bellman's principle of optimality, we need to take optimal decisions starting from the last reactor. The optimal decision time for each of the reactors is computed as well, starting from their respective expiration times and moving backwards in time to the initial state. The expiration time for ordering the $i$ th module is given by

$T_{i}=T_{n}-\sum_{k=i}^{n-1} C_{k}$

This constraint comes from the restriction that a new reactor can be ordered once all the prior ordered reactors have been constructed. Here $T_{n}$ is the expiration time for the option to start the construction of the last module and $C_{i}$ is the construction time in years for the $i$ th module.

At the expiration time for the last module the firm does not have the option to delay the investment. Therefore, the decision to start the construction is taken at those electricity prices for which the expected NPV of the last module is greater than zero. The option value of the last module at the expiration time is then given by:

$V_{n}\left(t_{m}=T_{n}, X_{t_{m}}\right)=\max \left(0, h_{n}\left(X_{t_{m}}\right)\right)$.

At time $t_{k}, k=m-1, \cdots, 0$, the option value for the last of the series of reactors is the maximum between immediate pay-off $h_{n}$ and its continuation value $Q_{n}$. The continuation value is the expected future cash flow if the decision to construct the reactor is delayed until the next time step. The reactor is constructed if at the given electricity price the net present value is greater than the expected cash flows if the reactor is constructed sometime in the future. This can be written as:

$V_{n}\left(t_{k}, X_{t_{k}}\right)=\max \left(h_{n}\left(X_{t_{k}}\right), Q_{n}\left(t_{k}, X_{t_{k}}\right)\right), k=0, \ldots, m-1$.

Given the present state $X_{t_{k}}$, the continuation value, or, in other words, the discounted cash flows if the decision to start the construction is delayed for the last reactor is,

$Q_{n}\left(t_{k}, X_{t_{k}}\right)=e^{-r\left(t_{k+1}-t_{k}\right)} \mathbb{E}\left[V_{n}\left(t_{k+1}, X_{t_{k+1}}\right) \mid X_{t_{k}}\right]$.

\footnotetext{
3 The optimal time to order is often called "optimal stopping time". In the case of sequential modular construction optimal stopping time would refer to the time when the option to delay the construction to the next time step terminates.
} 


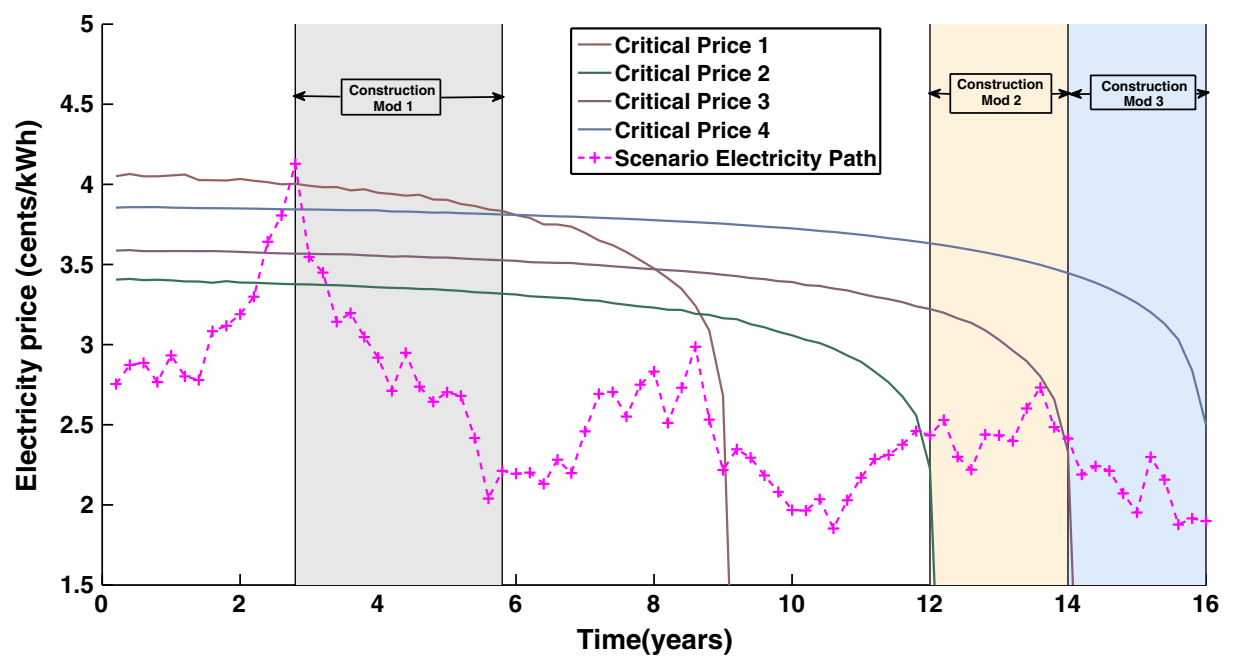

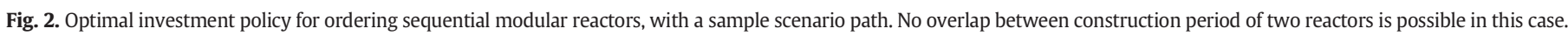

Once the option value at each time step for the last module is known, we move on to modules $n-1, \ldots, 1$. At the expiration time for the $i$-th module, the decision to start its construction is taken when the combined NPV of the present reactor and the expected future cash flow from the optimally constructed modules $i+1, \ldots, n$ is greater than zero. Therefore, the option value for the $i$ th module at its expiration time $T_{i}$ is given by:

$V_{i}\left(T_{i}, X_{T_{i}}\right)=\max \left(0, h_{i}\left(X_{T_{i}}\right)+Q_{i+1}\left(T_{i}, X_{T_{i}}\right)\right)$,

where $h_{i}$ gives the direct future cash flow from the $i$ th module and $Q_{i+1}\left(T_{i}, X_{T_{i}}\right)$ gives the expected cash flow from the optimal construction of modules $i+1, \ldots, n$, given the information $X_{T_{i}}$. The option value for the module at time step $t_{k}$, where $t_{k}<T_{i}$, is given by

$V_{i}\left(t_{k}, X_{t_{k}}\right)=\max \left(h_{i}\left(X_{t_{k}}\right)+Q_{i+1}\left(t_{k}, X_{t_{k}}\right), Q_{i}\left(t_{k}, X_{t_{k}}\right)\right)$,

i.e. the decision to start the construction of module $i$ is taken if the cash flow from its immediate construction (given by $h_{i}\left(X_{t_{k}}\right)$ ) and the expected cash flow from the modules $i+1, \ldots, n$, constructed optimally in the future (modeled by $Q_{i+1}\left(t_{k}, X_{t_{k}}\right)$ ), is greater than the expected cash flows from the modules $i, \ldots, n$, if the decision to start its construction is delayed to the next time step (given by $Q_{i}\left(t_{k}, X_{t_{k}}\right)$ ). The expected cash flow if the decision to start the construction of modules $i, \ldots, n$ is delayed to the next time step is given by:

$Q_{i}\left(t_{k}, X_{t_{k}}\right)=e^{-r\left(t_{k+1}-t_{k}\right)} \mathbb{E}\left[V_{i}\left(t_{k+1}, X_{t_{k+1}}\right) \mid X_{t_{k}}\right]$

The option value, $V_{i}\left(t_{k}, X_{t_{k}}\right)$, at time $t_{k}$ for constructing the module $i$ not only carries the information about the cash flows from module $i$, but also about the cash flows from the optimal construction of the modules $i+1, \ldots, n$ in the future.

For sequential modular construction the payoff for module $i$ is given by $h_{i}\left(X_{t_{k}}\right)+Q_{i+1}\left(t_{k}, X_{t_{k}}\right)$. The payoff does not only contain $h_{i}$, the direct discounted revenue from module $i$, but also $Q_{i+1}$, the value of the new option to start or delay the construction of new modules, that opens up with the construction of module $i$.

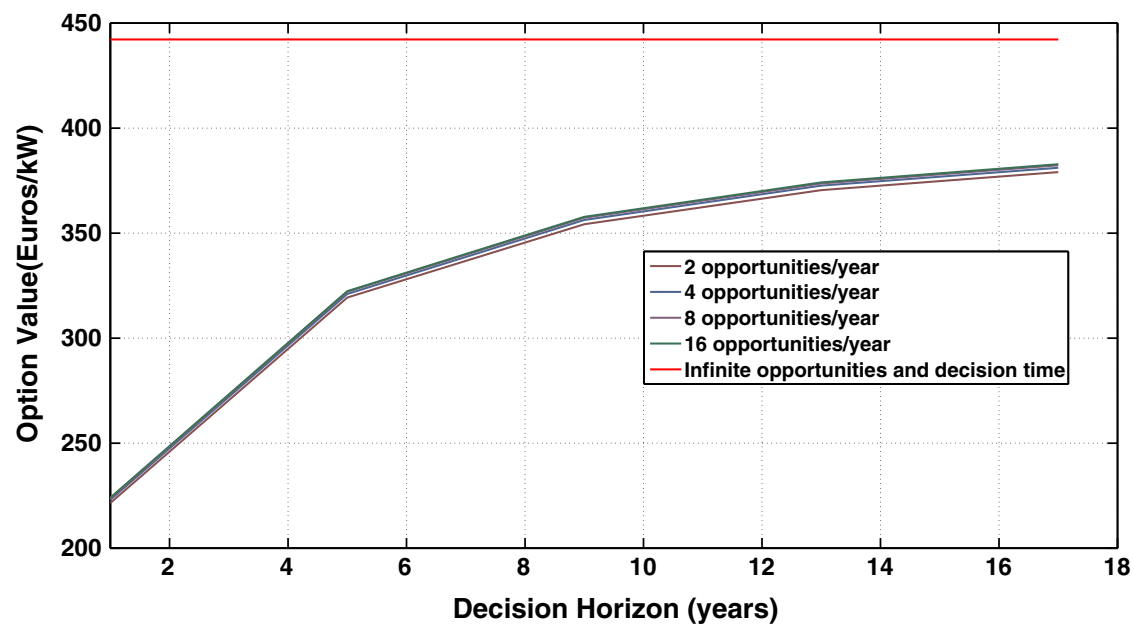

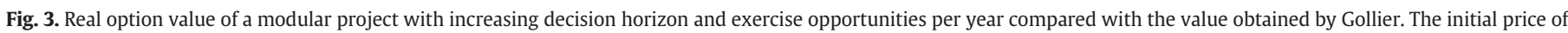
electricity is 3 cents $/ \mathrm{kWh}$ and volatility in electricity price is $20 \%$. 


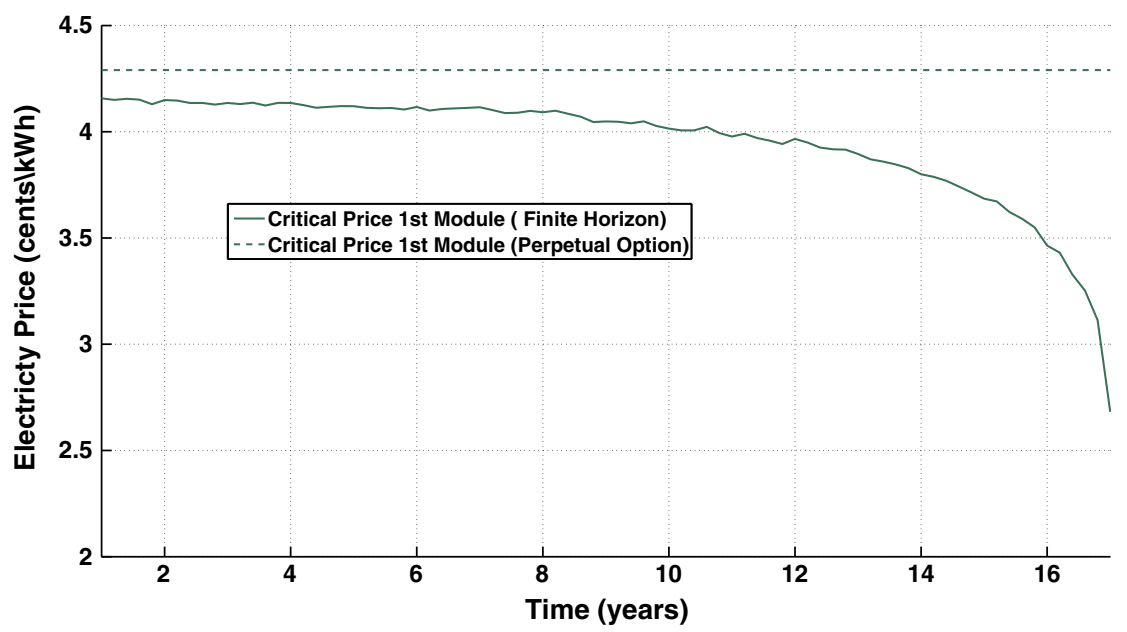

Fig. 4. Critical price at which the first module should be ordered, comparison between finite time and infinite time horizon.

\section{Stochastic grid method for multiple exercise options}

The real option problems we are interested in, have financial counterparts, i.e. the Bermudan options and multiple exercise Bermudan options. A Bermudan option gives the holder the right, but not obligation, to exercise the option once, on a discretely spaced set of exercise dates. A multiple exercise Bermudan option, on the other hand, can be exercised multiple times before the option expires. Pricing of Bermudan options, especially for multi-dimensional processes is a challenging problem owing to its path-dependent settings.

Consider an economy in discrete time defined up to a finite time horizon $T_{n}$. The market is defined by the filtered probability space $\left(\Omega, \mathcal{F}, \mathcal{F}_{t}, \mathbb{P}\right)$. Let $X_{t}$, with $t=t_{0}, t_{1}$, ots, $t_{m}=T_{n}$, be an $\mathbb{R}^{d}$-valued discrete time Markov chain describing the state of the economy, the price of the underlying assets and any other variables that affect the dynamics of the underlying. Here $\mathbb{P}$ is the risk neutral probability measure. The holder of the multiple exercise Bermudan option has $n$ exercise opportunities, that can be exercised at $t_{0}, t_{1}, \ldots, t_{m}$. Let $h_{i}\left(X_{t}\right)$ represent the payoff from the $i$ th exercise of the option at time $t$ and underlying state $X_{t}$. The time horizon for the $i$ th exercise opportunity is given by $T_{i}$.

We define a policy, $\pi$, as a set of stopping times $\tau_{n}, \ldots, \tau_{1}$ with $\tau_{n}<\ldots<\tau_{1}$, which takes values in $t_{0}, \ldots, t_{m}=T_{n}$, and $\tau_{i}$ determines the time where the ith remaining exercise opportunity can be used. The option value when there are $n$ early exercise opportunities remaining is then found by solving an optimization problem, i.e. to find the optimal exercise policy, $\pi$, for which the expected payoff is maximized. This can be written as:

$V_{n}\left(t_{0}, X_{t_{0}}=x\right)=\sup _{\pi} \mathbb{E}\left[\sum_{k=0}^{n} h_{k}\left(X_{\tau_{k}}\right) \mid X_{t_{0}}=x\right]$.

In simple terms, Eq. (13) states that of all possible policies for ordering the reactor in the given decision horizon, the real option value is computed using the one which maximizes the expected future cash flows.

In the past decade several simulation-based algorithms have been proposed for pricing Bermudan options. The regression based approach proposed by Carriere (1996), Tsitsiklis and Van Roy (1999) was popularized as the least squares method (LSM) by Longstaff and Schwartz (2001). Other important approaches include the stochastic mesh method of Broadie and Glasserman (2004), computing the early exercise frontier by Ibanez and Zapatero (2004) and the duality based method from Haugh and Kogan (2004) and Rogers (2002). More recently Jain

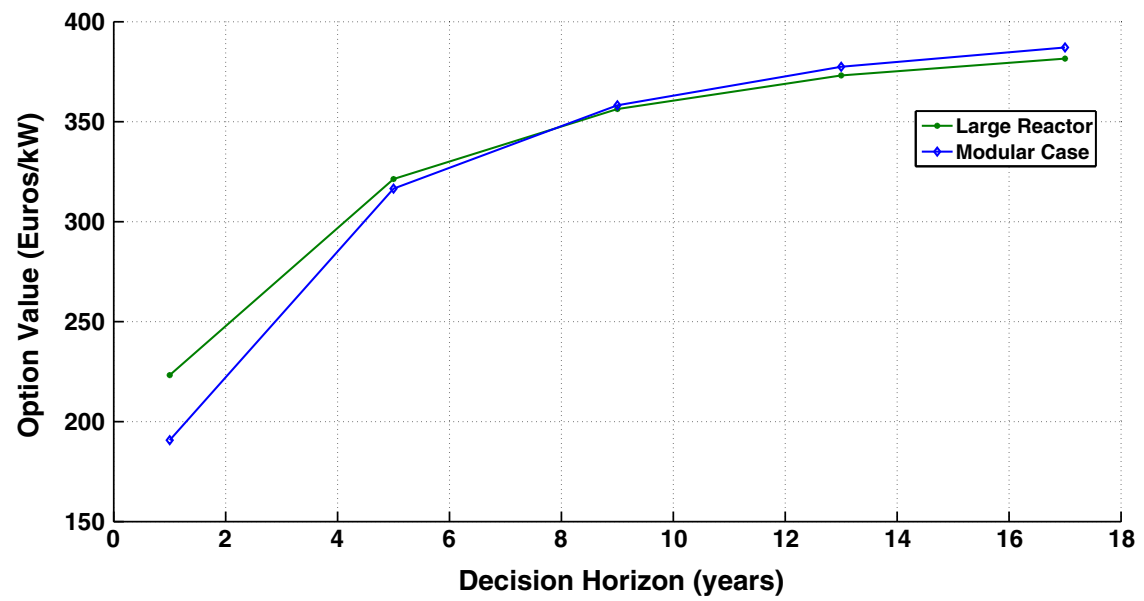

Fig. 5. Real option value for the large reactor and the modular project for different decision horizons when the initial price of electricity is 3 cents/kWh. 


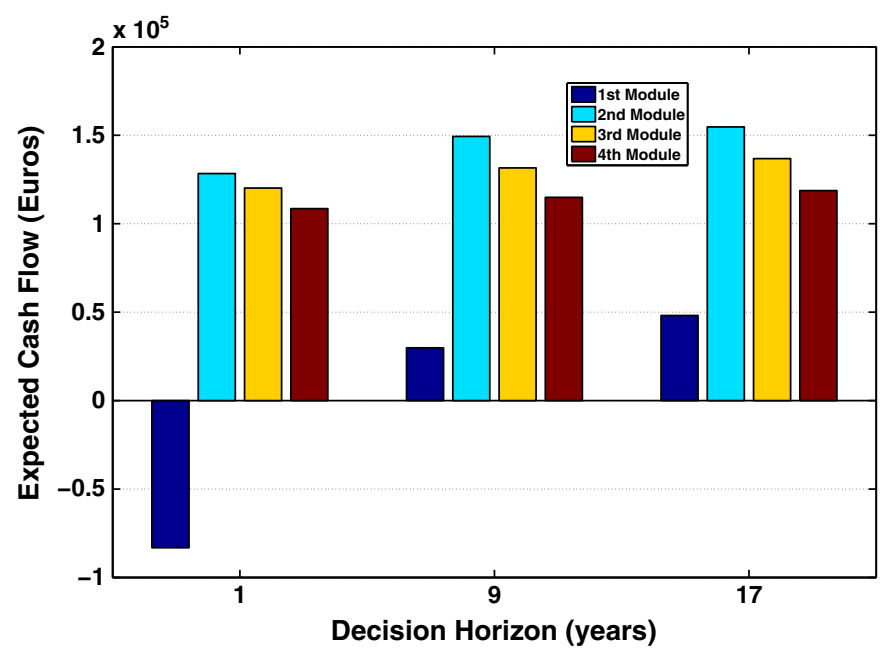

Fig. 6. Cash flow from different modules with increasing decision time. The initial price of electricity is 3 cents $/ \mathrm{kWh}$ and the volatility in electricity prices is $20 \%$.

and Oosterlee (2012) proposed the stochastic grid method (SGM) for pricing high-dimensional Bermudan options.

The problem of pricing Bermudan options with multiple exercise opportunities has been dealt with by Meinshausen and Hambly (2004), with generalizations by Bender (2009), Aleksandrov and Hambly (2008) and Schoenmakers (2012), who use the dual representation for such pricing problems. Chiara et al. (2007) apply the multiple exercise real options in infrastructure projects. They use a multi-least-squares Monte Carlo method for determining the option value.

The problem of sequential modular construction stated above can be solved using the stochastic grid method (Jain and Oosterlee, 2012). We choose the stochastic grid method, because:

- The stochastic grid method (SGM) can efficiently solve the multiple exercise Bermudan option problem;

- SGM can be used to compute the sensitivities of the real option value;

- The method can be easily extended to higher dimensions;

- The method doesn't depend on the choice of the underlying stochastic process;

- Improved confidence intervals are obtained with fewer paths when compared to LSM.

Although the problem we consider here is one-dimensional, with the electricity price as the stochastic variable, a typical real option problem tends to be high-dimensional with several underlying stochastic terms. A proper choice of pricing method would be one which can be extended to higher dimensions in the future.

The stochastic grid method solves a general optimal stopping time problem using a hybrid of dynamic programming and Monte Carlo simulation. The method first determines the optimal stopping policy and a direct estimator for the option price. The optimal stopping policy for the $i$ th module at time step $t_{k}$ involves finding the critical electricity price $X_{t_{k}}^{*}$. When the market price of electricity is equal to the critical price, the value of delaying the construction of the module to the next time step is equal to the value of starting the construction immediately, i.e.,

$Q_{i}\left(t_{k}, X_{t_{k}}^{*}\right)=h_{i}\left(X_{t_{k}}^{*}\right)$

Therefore, the critical price is taken to be the largest grid point $X_{t_{k}}$, for which $Q_{i}\left(t_{k}, X_{t_{k}}\right)>h_{i}\left(X_{t_{k}}\right)$. The module is ordered if the present market price of electricity is greater than the critical price for the given time step. Once the policy for all the time steps is known, SGM computes lower bound values, using a new set of simulated electricity paths, as the mean of the cashflows from each simulated path where the module is ordered following the policy obtained above.

SGM for multiple exercise Bermudan options begins by generating $N$ stochastic paths for the electricity prices, starting from initial state $X_{0}$. The electricity prices realized by these paths at time step $t_{k}$ constitute the grid points at $t_{k}$. The electricity price paths can be generated using Eq. (5) here.

The pricing steps for SGM can be decomposed into two main parts, based on the recursive dynamic programming algorithm from the previous section.

- Parametrization of the option value: The option values at each grid point are converted into a functional approximation using piece-wise regression.

- Computation of the continuation value: The continuation value is computed using the conditional probability density function and the functional approximation of the option value at the next time step.

\subsection{Parametrization of the option value}

In order to obtain the continuation value for grid points at $t_{k}$, we need to determine the functional approximations of the option value at $t_{k+1}$. Once the option values at the grid points at $t_{k+1}$ are known, the functional approximation is obtained using a piece-wise least squares regression. Therefore, the option value at a given time step is divided into two regions, separated by the critical electricity price $X_{t_{k+1}}^{*}$.

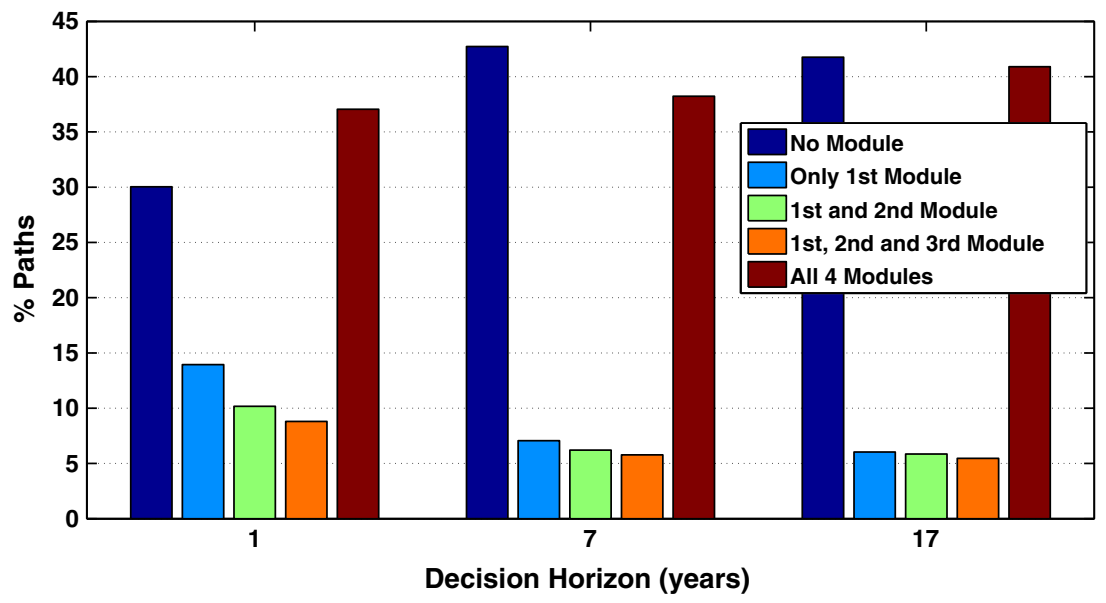

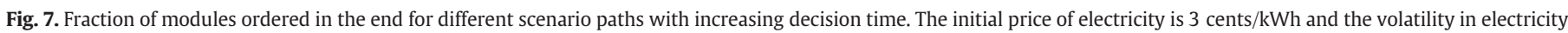
prices is $20 \%$. 


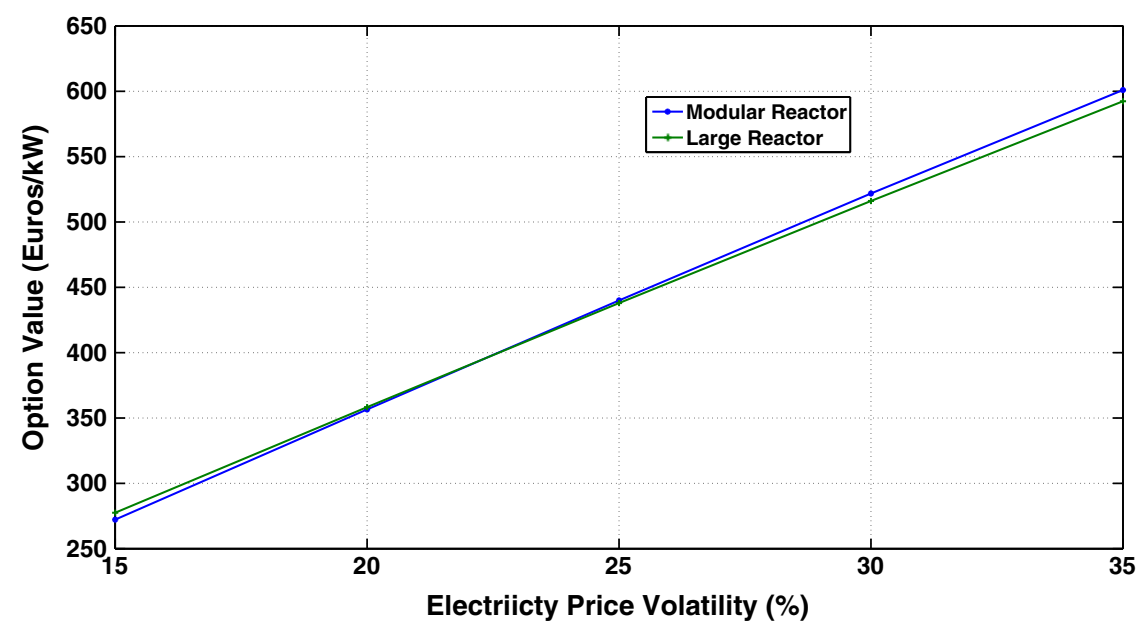

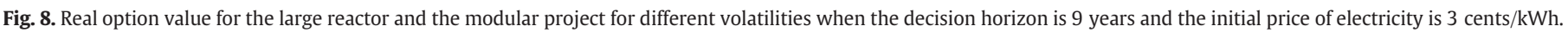

For the two segments the functional approximation is given by,

$$
\begin{aligned}
\hat{V}_{i}\left(t_{k+1}, X_{t_{k+1}}\right)= & { }^{1}\left\{X_{t_{k+1}}<X_{t_{k+1}}^{*}\right\} \sum_{m=0}^{M-1} a_{m} X_{t_{k+1}}^{m} \\
& +{ }^{1}\left\{X_{t_{k+1}} \geq \mathcal{X}_{t_{k+1}}^{*}\right\} \sum_{m=0}^{M-1} b_{m} X_{t_{k+1}}^{m} .
\end{aligned}
$$

The expression ${ }^{1}\left\{X_{t_{k+1}}<\mathcal{X}_{t_{k+1}}^{*}\right\}$ is an indicator function whose value equals 1 , if the argument $\left\{X_{t_{k+1}}<\mathcal{X}_{t_{k+1}}^{*}\right\}$, is true and it is 0 otherwise. Therefore, ${ }^{1}\left\{X_{t_{k+1}}<\mathcal{X}_{t_{k+1}}^{*}\right\}$ and ${ }^{1}\left\{X_{t_{k+1}} \geq \mathcal{X}_{t_{k+1}}^{*}\right\}$ group the grid points into two segments, separated by the critical electricity price. The results converge to the true price when increasing number of segments are used (see Jain and Oosterlee, 2012).

\subsection{Computation of the continuation value}

Once the functional approximations of the option values for modules $i$ and $(i+1)$ are known for time step $t_{k+1}$, the continuation value for the ith module at $t_{k}$ can be computed using Eq. (12). In order to compute the expectation, $\mathbb{E}\left[V_{i}\left(t_{k+1}, X_{t_{k+1}}\right) \mid X_{t_{k}}\right]$, we need the distribution function for $X_{t_{k+1}}$ given $X_{t_{k}}$. This conditional distribution function, $f\left(X_{t_{k+1}} \mid X_{t_{k}}=x\right)$, for the GBM process is known in closed form.
Therefore, the continuation value, or the value of the reactor if the decision to order it is delayed to the next time step, as given Eq. (12) can be written as:

$$
\begin{aligned}
\hat{Q}_{i}\left(t_{k}, X_{t_{k}}\right)= & \left.\int_{y \in\left[0, \mathcal{X}^{*}\right]}\left(\sum_{m=0}^{M-1} a_{m} y^{m}\right) f\left(y \mid X_{t_{k}}=x\right) d y\right) \\
& +\int_{y \in\left(\mathcal{X}^{*}, \infty\right]}\left(\sum_{m=0}^{M-1} b_{m} y^{m}\right) f\left(y \mid X_{t_{k}}=x\right.
\end{aligned}
$$

In a more generic case where the conditional distribution function is unknown, it can be approximated using the Gram Charlier Series. For more details on computing the continuation value, we refer to (Jain and Oosterlee, 2012).

\section{Numerical experiments}

We consider the case where an investor needs to decide between two projects, one involving a single large reactor of 1200 MWe and the other consisting of four modules of 300 MWe each. The construction time and costs for the two projects, given in Table 1, are taken from the reference case by Gollier et al. (2005). The discount rate is taken as $8 \%$ per annum, which is the OECD average, and the predicted growth rate of electricity price is $0 \%$ here. The cost of electricity production for the first unit is relatively expensive when compared to series units, as a large part of the fixed costs for the modular assembly,

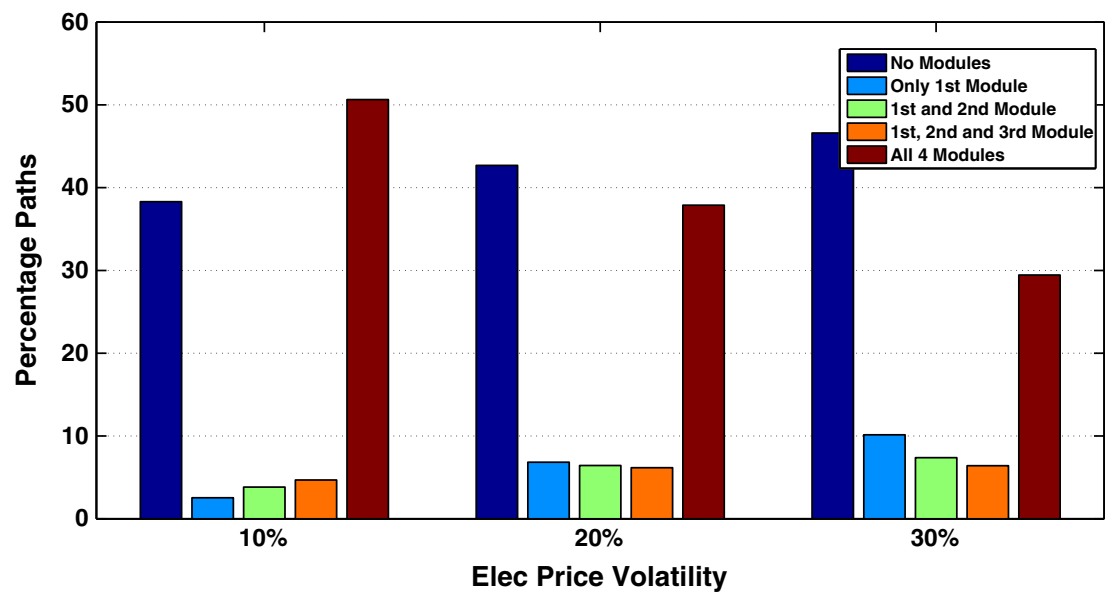

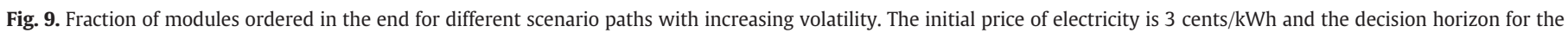
first module is 9 years. 
like the land rights, access by road and railway, site licensing cost, are connection to the electricity grid are carried by the first unit.

In the case of the modular project we consider two different constraints, in two subsections to follow, i.e., the decision for construction of subsequent units can be made:

1. Once the construction of all prior units is completed (similar to the case considered by Gollier),

2. Once the decision for the construction of all prior units has been taken. Also, only one unit can be ordered at a given time step.

\subsection{Sequential construction: the case in Gollier et al. (2005)}

In this test case we apply constraint 1 for the construction of subsequent modules, i.e., the decision for the construction of a new module will not be made, unless the construction of all previous modules is finalized. By the SGM we first obtain an optimal investment policy and a direct estimator of the real option value of the project. The optimal policy gives the critical electricity price (as a function of time), above which a module should be ordered. At a given time a new module is ordered only when the present electricity is higher than the corresponding critical price for the module under consideration and when all other constraints are satisfied. Once the optimal investment policy is obtained, a fresh set of electricity paths is generated, and at each of these simulated paths a new module is decided if the following conditions are satisfied:

1. All modules preceding the given module have been constructed.

2. The present electricity price is higher than critical price for ordering the given module.

3. The present time is within the decision horizon for the corresponding module.

4. The given module hasn't been ordered so far for the given path.

Fig. 2 illustrates for a sample electricity path when a new module should be ordered. A module is ordered once the above conditions are satisfied and the revenue from this module is discounted back to the initial time. The mean of the discounted revenue for different paths from all the four modules gives the real option value of the project. For a single large reactor the steps followed are the same, except that condition 1 is not required.

As the case in Gollier et al. (2005) corresponds to an infinite horizon decision problem with exercise opportunities, we compare it with an increasing finite time decision horizon. Fig. 3 compares the

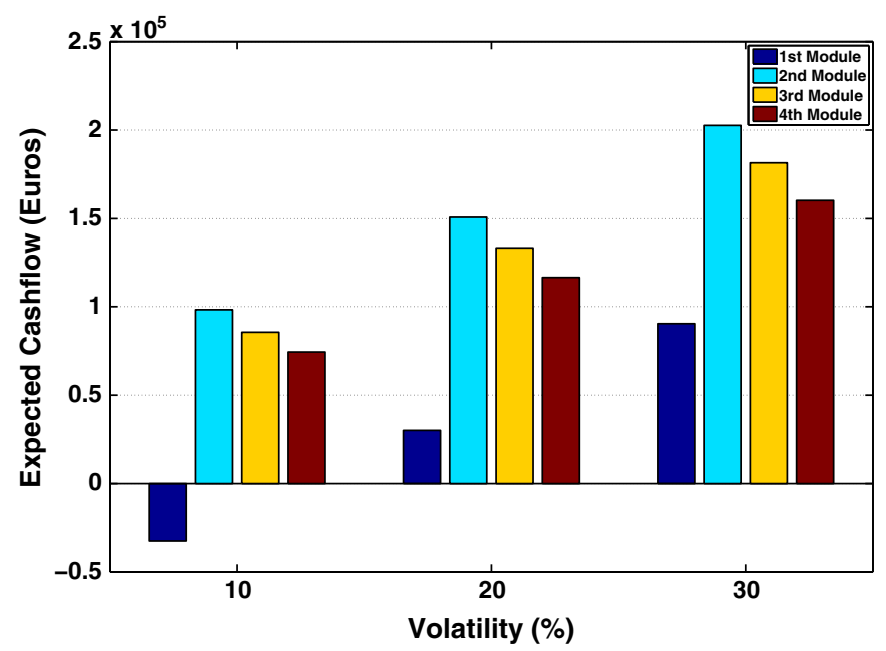

Fig. 10. Expected cashflow from different units for different volatility values for electricity prices. The initial price of electricity is 3 cents/kWh and the decision horizon for the first module is 9 years.

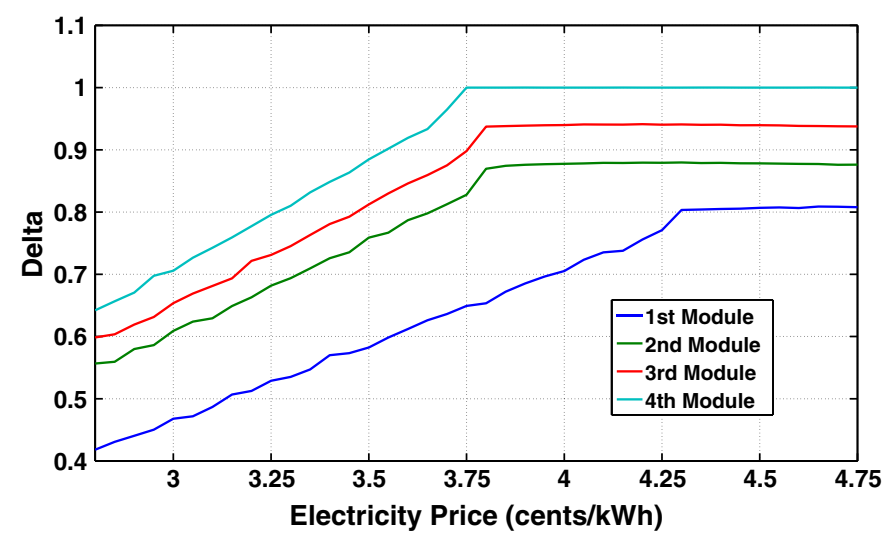

Fig. 11. The delta values for the four modules when the decision horizon is 12 years and the volatility in electricity price is $20 \%$.

real option value of the modular project with the reference value in Gollier et al. (2005). The option value of the project doesn't increase much with an increasing number of exercise opportunities per year, however it increases significantly with an increasing decision horizon. From Fig. 3 it's clear that the real option value of a modular project with a realistic decision horizon is lower than the value obtained in Gollier et al. (2005), where an infinite decision horizon is assumed. In other simulations, not reported here, we found that the option value of the modular project with the same parameters, but with a decision horizon of 100 and 200 years and four exercise opportunities per year, has an option value between 390 and 400 Euro/kW, which is already closer to the infinite horizon values.

Fig. 4 then compares the optimal investment policy for the first module with the corresponding policy in Gollier et al. (2005). It shows clearly the effect of a finite decision horizon on the investment policy. As one approaches the final decision time, the value of waiting (given by the continuation value) reduces which lowers the threshold electricity price at which a new module should be ordered. However, in the case of an infinite decision time horizon, the optimal policy or threshold electricity price remains constant with time.

\subsubsection{Comparison of two projects with different decision times}

We now compare the real option values of the two projects, i.e. the single large reactor and the sequence of small modular units, for increasing decision time horizon and uncertainty in electricity prices. The construction costs and times for the reactors are taken from Table 1. Based on Eq. (6), we take the corresponding decision horizon for the construction of the first module between 1 and 17 years. For the single large reactor we take the decision horizon the same as that for first module. One of the advantages of a modular construction is that the increasing demand can be met gradually, which allows the spreading of the decisions to a longer time horizon, possibly without significant gaps in

Table 2

Critical threshold electricity prices (cents/KWh) at which new reactors should be ordered, for different decision horizons. There are twenty equally spaced exercise opportunities each year. The volatility of the electricity price is taken as $20 \%$.

\begin{tabular}{|c|c|c|c|c|c|c|c|}
\hline & \multirow{2}{*}{$\frac{\text { Final decision }}{\text { Time (years) }}$} & \multicolumn{2}{|c|}{ Isolated } & \multicolumn{4}{|c|}{ Modular } \\
\hline & & LR & Unit 1 & Unit 1 & Unit2 & Unit3 & Unit 4 \\
\hline \multirow[t]{3}{*}{ SGM } & 12 & 4.56 & 5.98 & 4.05 & 3.44 & 3.65 & 3.93 \\
\hline & 17 & 4.60 & 6.02 & 4.18 & 3.50 & 3.69 & 3.96 \\
\hline & 22 & 4.62 & 6.05 & 4.24 & 3.53 & 3.71 & 3.98 \\
\hline \multirow[t]{3}{*}{$\cos$} & 12 & 4.56 & 5.98 & 4.10 & 3.46 & 3.65 & 3.93 \\
\hline & 17 & 4.60 & 6.03 & 4.21 & 3.51 & 3.69 & 3.96 \\
\hline & 22 & 4.62 & 6.05 & 4.25 & 3.53 & 3.71 & 3.98 \\
\hline Gollier & $\infty$ & 4.75 & 6.23 & 4.29 & 3.57 & 3.79 & 4.10 \\
\hline
\end{tabular}




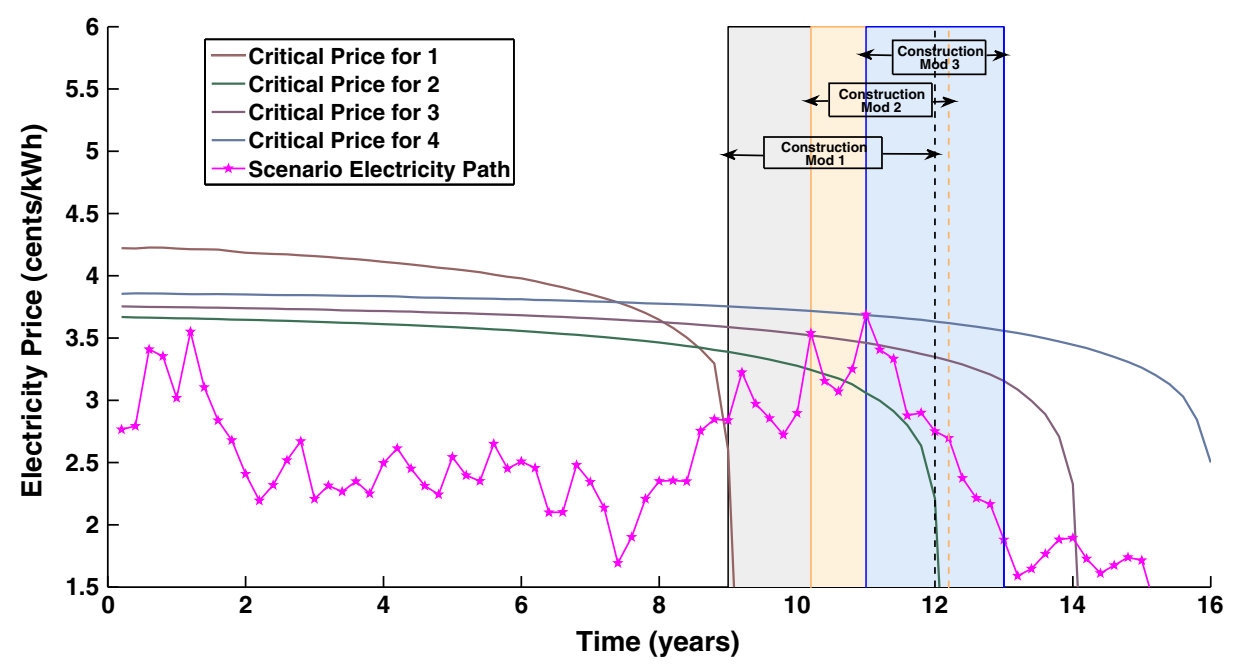

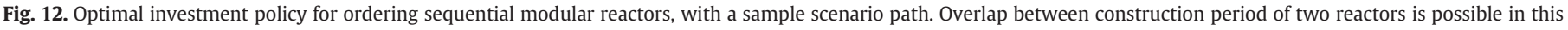
case.

demand and supply. Therefore, although the decision horizon for the first module can be small, the decision horizon for the entire project can be longer.

Fig. 5 compares the option value for the single large reactor with that for the modular project for increasing decision time. When the decision horizon is small it is optimal to opt for the large reactor whereas for longer decision horizons modular projects appear more profitable.

An insight into the reason why modular projects are better for longer decision time is given by Fig. 6, which shows the expected cashflow from the four units for increasing decision time. When the decision horizon is small, the expected cashflow from the first module can be negative, which is the case when the decision horizon for the first module is 1 year. For short decision times, the first unit needs to be ordered to keep open the option to order more profitable subsequent modules. As the time approaches the final decision time for the first unit, it can be ordered even if the expected revenue from its construction would be negative. With increasing decision horizon the investor can wait longer and order the modules at more profitable electricity prices.

Fig. 7 shows fractions of the scenario paths for which different numbers of modules are ordered. When the decision horizon is small, the project is only partially completed for a large number of scenario paths, making it unprofitable. However, for longer decision times, the fraction of the scenario paths for which all the four modules are constructed increases, while the fraction of partially completed project reduces.

5.1.2. Comparison of two projects and different volatilities in electricity prices

A parameter to be considered when deciding between a single large reactor and the modular project is the uncertainty in electricity prices. We compare the real option value of the two projects for increasing volatility in the electricity price in Fig. 8.

When the volatility in the electricity price is low, the single large reactor project is more profitable, while for higher volatilities the modular project seems a better choice. An intuitive answer to this is that, for high volatilities, modular projects offer more flexibility, i.e., if the electricity price path at some point reaches unfavorable prices, the possibility to abandon module construction, with a few units already ordered, exists. Fig. 9 shows the fraction of scenario paths for which the modular project finishes with different numbers of units ordered. As expected, the fraction of paths for which not all the four units are ordered increases with increasing volatility.

Fig. 10 gives the expected cashflow from each unit for different volatilities of the electricity prices. In general, the option value of the project increases with an increasing uncertainty. A higher volatility reflects greater future price fluctuations (in either direction) in underlying electricity price levels. This expectation generally results

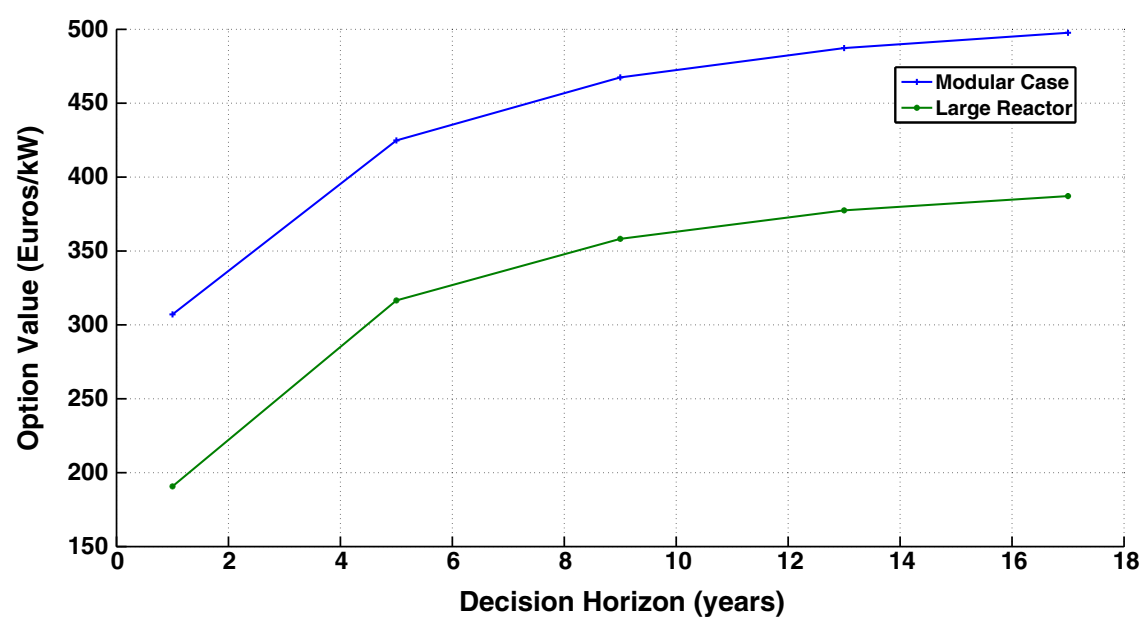

Fig. 13. Real option value for the large reactor and the modular project for different decision horizons when the initial price of electricity is 3 cents/kWh. 


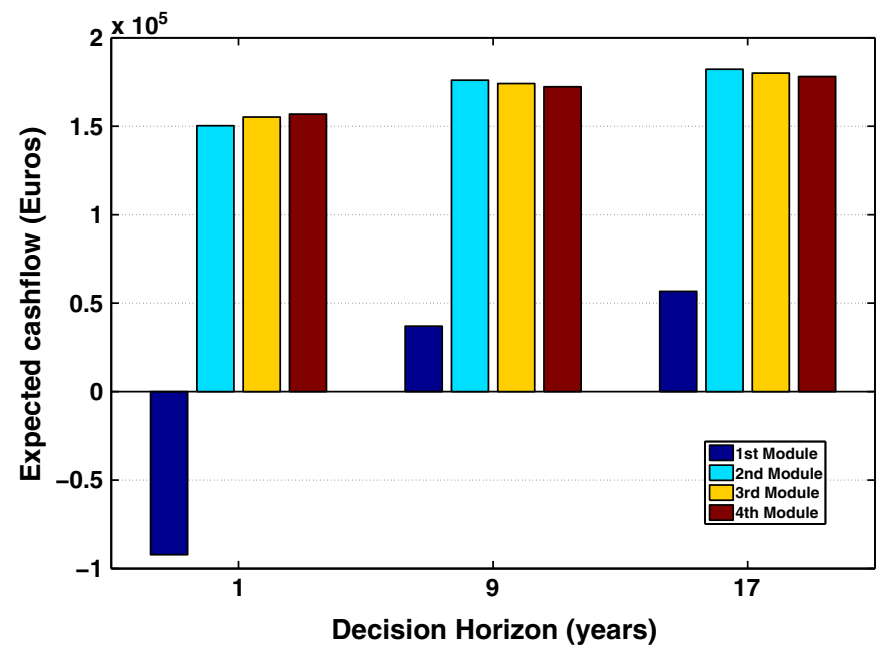

Fig. 14. Cash flow from different modules with increasing decision time. The initial price of electricity is 3 cents/kWh and volatility in electricity prices is $20 \%$.

in a higher option premium, especially if the option is exercised optimally. The cashflow from the first unit is smallest because it's the most expensive of the four units. When the cost of the units would be same, as is the case for units 2 to 4 , the discount factor plays an important role, making the present value of units that are ordered first larger than the value from units further in the future.

For a firm it is not only important to know the real option value for making an investment decision, but also the sensitivity ${ }^{4}$ of the value with respect to the parameters chosen. We here compute the delta values, i.e. the ratios of the change in the real option value of the reactors to the change in the underlying electricity price. High delta values imply that the investment decisions are sensitive to a changing electricity price. Fig. 11 compares the delta values for the different modules. We see that the delta values for the final module, as expected, converge to one, i.e. when it's optimal to order a new reactor the change in option value is proportional to the change in the electricity price. However, for each prior module the delta values converge to values less than one. For the first module a unit change in the electricity price changes the option price by a factor of 0.8 . This makes the modular construction investment option generally more stable, i.e. even if the electricity price drops by 1 the value of investment changes only by a factor of 0.8 .

Table 2 gives the critical threshold prices for constructing a single large reactor and for the different modules. We compare the SGM results with those computed using the $\operatorname{COS}$ method $^{5}$ (Fang and Oosterlee, 2008) and with Gollier et al. (2005). The COS method is a deterministic method, developed for computational finance applications, which can easily be applied to sequential investment decisions for single "assets". The Monte Carlo and the COS method give identical prices, which is a validation for the MC method, and we see a clear difference between the results obtained for finite time and those for infinite time horizon decisions in Gollier et al. (2005). From these results we can conclude that SGM is a good candidate for pricing finite time real option problems, as it can also be extended to higher dimensions in a straightforward way.

\subsection{Modified case: multiple construction, sequential ordering}

In this section we consider the two projects discussed above, except that we now relax one of the constraints in the case of the

\footnotetext{
${ }^{4}$ Sensitivity analysis in financial options is performed by computing the derivatives with respect to various parameters, and these derivatives together are referred to as the Greeks.

${ }^{5}$ We would like to thank Marjon Ruijter for the COS method results.
}

modular project, i.e., a new unit can be ordered if all previous units have been ordered (not constructed). Only a single unit can be ordered at any given time step. It's common practice to have parallel construction of different units in order to achieve cost savings, as it allows rotation of specialized labor between different units (NEA, 2000).

Fig. 12 shows a scenario path and investment policy for a modular project with the above considerations. It can be seen that in this case overlap in the construction period of different units is possible.

\subsubsection{Comparison of two projects and different decision times}

Fig. 13 compares the real option values of the two projects for different decision times. The decision time for the large unit is kept the same as that for the first unit in the modular project. As mentioned before, decisions of generation capacity expansion are based on meeting increasing electricity demands with a certain minimum reliability and therefore the decision horizon is chosen to be the same for the first module and the large reactor. In this case the modular project appears more profitable than the single large reactor.

In order to detail the results obtained, we compute the expected cashflow from different units of the modular project and the fraction of modules ordered for different decision times. Fig. 14 gives the expected cashflow from the four units for different decision times. It can be seen that the expected cashflow grows with decision time. When overlap in the construction periods of different units is allowed, the cashflow from the three similar costing units is almost the same. The reason for this is that most often the three units are ordered around the same time, and so the effect of discounting to present time is almost the same. An important reason for modular projects having higher real option value is that the effective decision horizon for the modular project is significantly longer than that of the large reactor (which is the same as that of the first unit). Another factor which adds up to the profitability of the modular project (when parallel construction is allowed) is that modular units have less construction time, which allows cashflow from the sale of electricity to start before it would start from the large reactor.

Fig. 15 shows the fraction of different modules constructed by the end of the decision time for the modular project. It is clear that when the constraint of waiting for completion of a unit before ordering a new one is relaxed, that once the first unit is ordered, in most cases it results in all four units being ordered.

\subsubsection{Comparison of two projects for different electricity price volatilities}

Fig. 16 compares the real option values of the two projects for different volatilities in electricity prices under the relaxed constraint. We see in this case that the modular project is always more profitable than a single large reactor.

When the constraint of ordering a new unit is relaxed from waiting until completion of all previous units to waiting until ordering of all previous units, most of the units are ordered around the same time, as can be concluded from the discussion above. However, this will not be the case when the uncertainty in the electricity price increases. Fig. 17 shows the fraction of scenario paths for which different numbers of units are ordered by the end of the decision horizon. It is clear that with increasing uncertainty more often the project ends with fewer units than were planned initially.

The cashflow for the project from different units however increases with increasing uncertainty in electricity prices, as can be seen in Fig. 18.

\section{Conclusions}

In this paper we present a flexible and accurate valuation method for computing real option values, and critical electricity prices, related to the construction of nuclear power plants. We have developed a Monte Carlo based method, called the stochastic grid method (SGM), to analyze some scenarios of interest for a utility when choosing nuclear reactors. In 


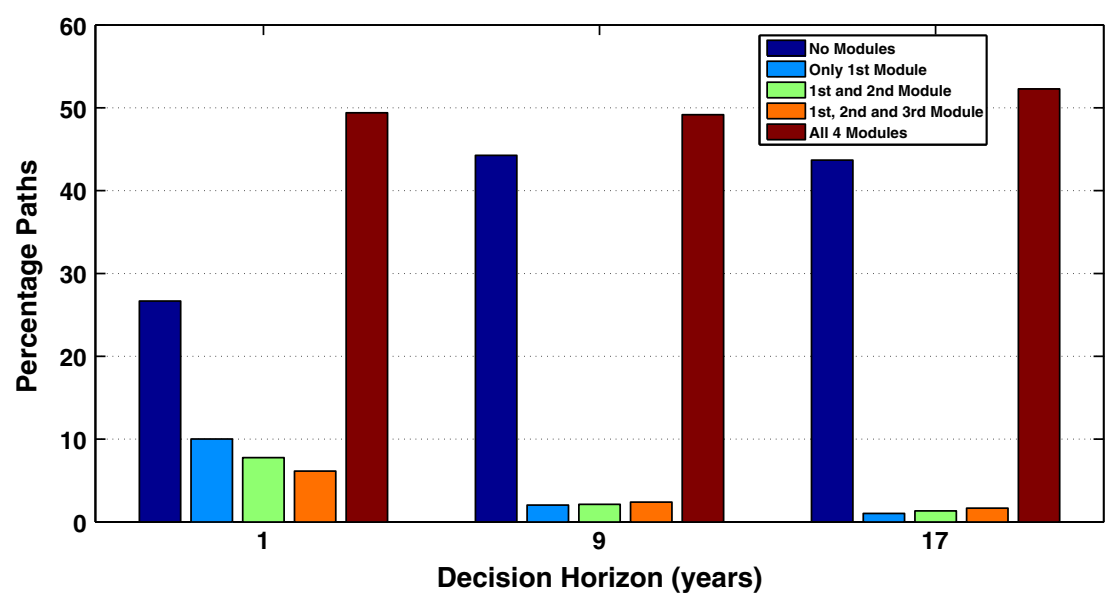

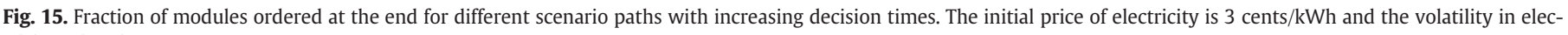
tricity prices is $20 \%$.

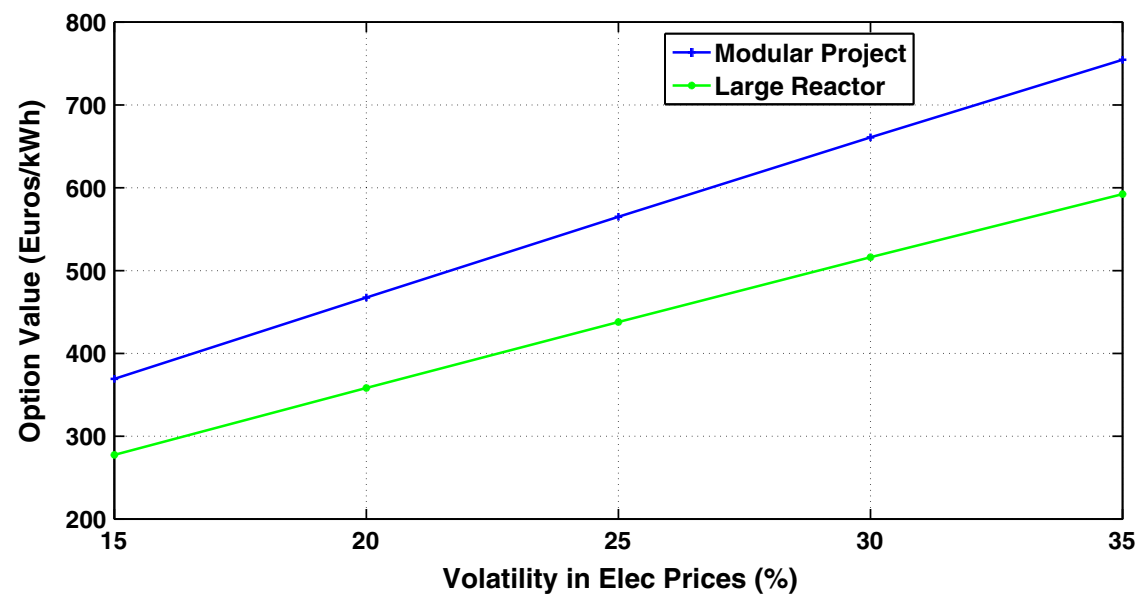

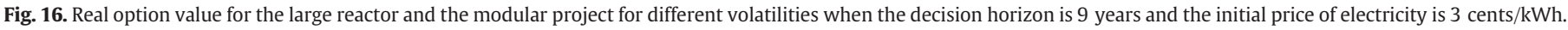

particular, we have focused our attention to the modular construction and finite decision horizons.

Some of the outcomes can be summarized as:

- SGM is a suitable Monte Carlo method for pricing real options, especially for valuing projects with modularity. The method has been validated against the deterministic COS method for a 1D test case.

- Most investment decision problems are governed by a finite decision time. It has been shown, in various numerical experiments, that decision-making in finite time may result in quite different scenarios compared to infinite time decision problems.

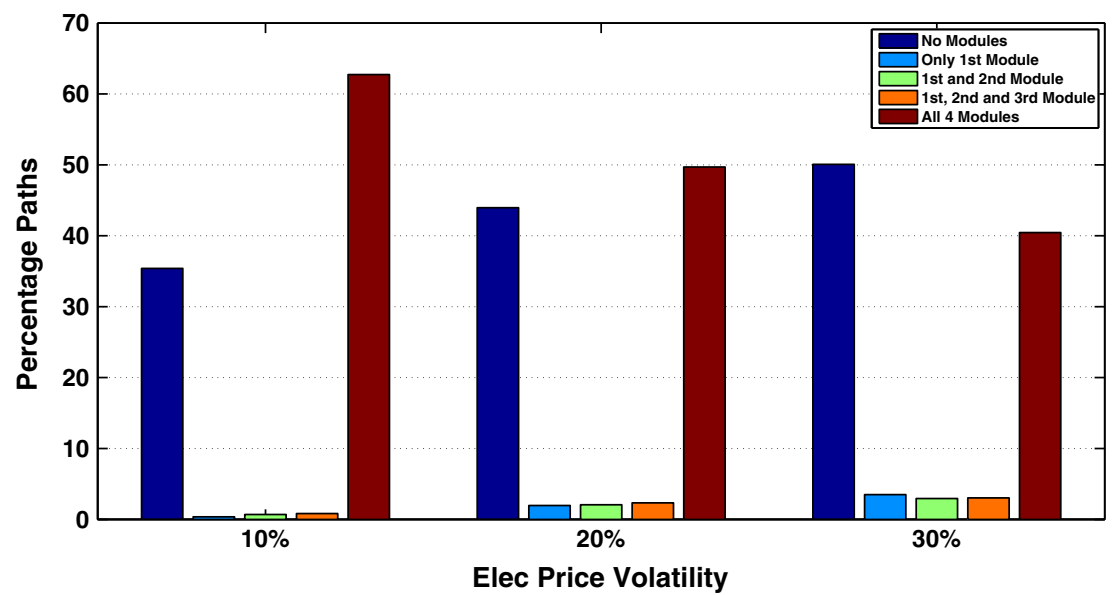

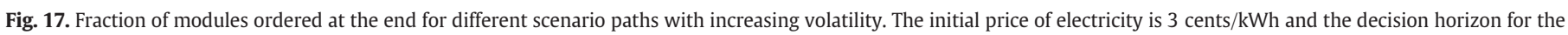
first module is 9 years. 


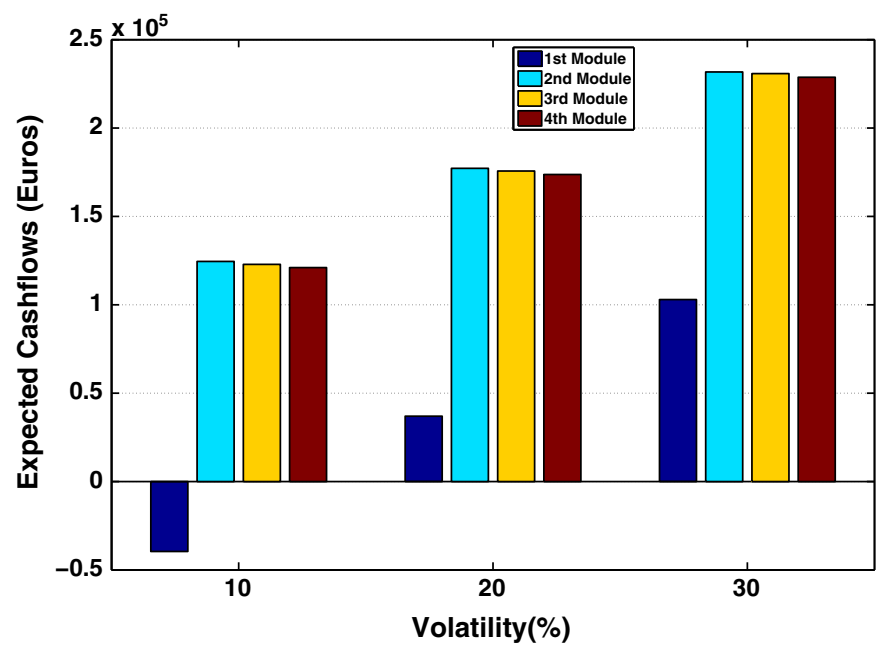

Fig. 18. Expected cashflow from different units for different volatilities of electricity prices. The initial price of electricity is 3 cents/kWh and the decision horizon for the first module is 9 years.

- When the modular project has a restriction on parallel construction of different units then:

- The real option value of a single large reactor is typically higher when decision horizons are small.

- For longer decision times modular projects may be more profitable.

- With an increasing uncertainty in the electricity prices, modeled by a higher volatility in the chosen electricity price model, modular construction may represent a better option. Modular construction may include the possibility of having a few units ordered, when the electricity price reaches unfavorable values. With stable electricity prices the cost effective single large reactor appears to be a better choice.

- When there is the possibility of parallel construction of modular units, then:

- the option value of the modular project greatly improves, and seems, with our model assumptions, more profitable than a single large reactor for different decision horizons.

- For different electricity price volatility values, the modular project seems to be the better choice.

- In many cases, once the first unit of the modular project is ordered it results in all units being ordered.

- The option value of the modular project greatly improves, and seems, with our model assumptions, more profitable than a single large reactor for different decision horizons.

- For different electricity price volatility values, the modular project seems to be the better choice.

- In many cases, once the first unit of the modular project is ordered it results in all units being ordered.

This paper serves as a validation of our method against the results obtained in Gollier et al. (2005). In our future research, a more detailed analysis may include a sophisticated electricity price model, demand and capacity factors, and stochastic construction and operation costs. In a follow-up paper we also discuss the impact of features like the construction of twin reactors (parallel construction of modules), effect of learning and rare events on the real option values of various scenarios.

It's worth noting that the real option values computed here does not include electricity price prediction model uncertainty, which is typically called model risk in finance. We presume here that the electricity price follows GBM and the scenario paths used for calculations follow the same model. In reality however, the model presumed by a firm wouldn't exactly replicate the actual electricity price process distribution. Model risk can however be assessed by varying the price dynamics and studying the impact on the real option values. Furthermore, one can always compute sensitivities with respect to the different problem parameters. In the present paper however, the purpose of the study was to compare two projects in which case the electricity price prediction model uncertainty effects the valuation of both of them similarly.

\section{References}

Aleksandrov, N., Hambly, B.M., 2008. A dual approach to multiple exercise option problems under constraints. Math. Meth. Oper. Res. 71 (3), 503-533.

Arrow, K.J., Fischer, A.C., 1974. Environmental preservation, uncertainty and irreversibility. Q. J. Econ. 88, 312-319.

Barlow, M., 2002. A diffusion model for electricity prices. Math. Finance 12 (4), 287-298.

Bender, C., 2009. Dual pricing of multi exercise options under volume constraints. Finance Stoch. 15 (1), 1-26.

Boarin, S., Locatelli, G., Mancini, M., Ricotti, E., 2012. Financial case studies on small and modular reactors. Nucl. Technol. 178.

Brennan, M.J., Schwartz, E.S., 1985. Evaluating natural resource investments. J. Bus. 58, 135-157.

Broadie, M., Glasserman, P., 2004. A stochastic mesh method for pricing high-dimensional American option. J. Comput. Finance 7, 35-72.

Carelli, M.D., Garrone, P., Locatelli, G., Mancini, M., Mycoff, C., Trucco, P., Ricotti, M.E. 2010. Economic features of integral, modular, small-to-medium size reactors. Prog. Nucl. Energy 52, 403-414.

Carriere, J.F., 1996. Valuation of the early exercise price for derivative securities using simulation and splines. Insurance: Mathematics and Economics 19, 19-30.

Chiara, N., Garvin, M.J., Vecer, J., 2007. Valuing simple multiple-exercise real options in infrastructure projects. J. Infrastruct. Syst. 13 (2), 97-104.

Dixit, A.K., Pindyck, R.S., 1994. Investment Under Uncertainty. Princeton University Press, Princeton.

Fang, F., Oosterlee, C.W., 2008. A novel pricing method for European options based on Fourier-cosine series expansions. SIAM J. Sci. Comput. 31, 826-848.

Gollier, C., Proult, D., Thais, F., Walgenwitz, G., 2005. Choice of nuclear power investments under price uncertainty: valuing modularity. Energy Econ. 27, 667-685.

Haugh, M., Kogan, L., 2004. Approximating pricing and exercising of high-dimensional American options: a duality approach. Oper. Res. 52 (2), 258-270.

Henry, C., 1974. Investment decisions under uncertainty: the irreversibility effect. Am. Econ. Rev. 64, 1006-1012.

Ibanez, A., Zapatero, F., 2004. Monte Carlo valuation of American options through computation of the optimal exercise frontier. J. Financ. Quant. Anal. 39 (2), 253-275.

Jain, S., Oosterlee, C.W., 2012. Pricing high-dimensional Bermudan options using the stochastic grid method. Int. J. Comput. Math. 89 (9), 1186-1211.

Kessides, I.N., 2010. Nuclear power: understanding the economic risks and uncertainties. Energy Policy 38, 3849-3864.

Longstaff, F.A., Schwartz, E.S., 2001. Valuing American options by simulation: a simple least-squares approach. Rev. Financ. Stud. 3, 113-147.

Lucia, J.J., Schwartz, E.S., 2002. Electricity prices and power derivatives: evidence from the Nordic power exchange. Rev. Deriv. Res. 5 (1), 5-50.

McDonald, R., Siegel, D., 1986. The value of waiting to invest. Q. J. Econ. 101, 707-727.

Meinshausen, N., Hambly, B.M., 2004. Monte Carlo methods for the valuation of multiexercise options. Math. Finance 14 (4), 557-583.

NEA, 2000. Reduction of Capital Costs of Nuclear Power Plants. OECD/NEA.

Paddock, J.L., Siegel, D.R., Smith, J.L., 1988. Option valuation of claims on real assets: The case of offshore petroleum leases. The Quarterly Journal of Economics 103 (3), 479-508.

Pilipovic, D., 1998. Energy Risk: Valuing and Managing Energy Derivatives. McGrawHill Publishers.

Pindyck, R.S., 1993. Investments of uncertain costs. J. Financ. Econ. 34, 53-76.

Rogers, L.C.G., 2002. Monte Carlo valuation of American options. Math. Finance 12, $271-286$.

Rothwell, G., 2006. A real options approach to evaluating new nuclear plants. Energy J. 27 (1), 37-53.

Schoenmakers, J., 2012. A pure martingale dual for multiple stopping. Finance and Stochastics 16 (2), 319-334.

Trigeorgis, L., 1996. Real Options: Managerial Flexibility and Strategy in Resource Allocation. MIT Press, Cambridge, MA.

Tsitsiklis, J., Van Roy, B., 1999. Optimal stopping of Markov processes: Hilbert space theory, approximation algorithms, and an application to pricing high-dimensional financial derivatives. IEEE Transactions on Automatic Control 44, 1840-1851. 